\title{
Circuit Modeling and Signal Transmission Analysis of Inductive Coupler in Wired Drill Pipe
}

\author{
Jia Jia, ${ }^{1}$ Yinao Su $\mathbb{D}^{1,2}$ Yue Shen $\mathbb{D}^{3},{ }^{3}$ Long Wang, ${ }^{3}$ Lingtan Zhang, ${ }^{3}$ and Yan Ling ${ }^{3}$ \\ ${ }^{1}$ China University of Petroleum (East China), School of Petroleum Engineering, Qingdao 266580, China \\ ${ }^{2}$ CNPC Engineering Technology R\&D Company Limited, Beijing 102206, China \\ ${ }^{3}$ China University of Petroleum (East China), College of Science, Qingdao 266580, China
}

Correspondence should be addressed to Yinao Su; suyinao1949@163.com

Received 21 January 2021; Revised 9 February 2021; Accepted 17 March 2021; Published 27 March 2021

Academic Editor: Salman Saleem

Copyright ( 92021 Jia Jia et al. This is an open access article distributed under the Creative Commons Attribution License, which permits unrestricted use, distribution, and reproduction in any medium, provided the original work is properly cited.

\begin{abstract}
The telemetry drill string transmits the measured drilling and formation parameters near the bit to the ground at high speed through the transmission line in the wired drill pipe and the inductive coupler formed by the coil embedded in the drill pipe joint. The voltage transfer function of the inductive coupler seriously affects the signal transmission characteristics of the telemetry drill string. Present research methods cannot accurately and quantitatively describe the influence of the electromagnetic parameters of the inductive coupler on its voltage transfer function. This paper quantitatively analyzes the effects of the electromagnetic parameters of the inductive coupler on signal transmission through reasonable high-frequency circuit modeling. The generation mechanism of eddy current in the magnetic core and the interaction mechanism between eddy current and coupling coils are studied using the electromagnetic detection analysis method of nondestructive testing (NDT) technology. Then, the highfrequency circuit model of the coupler containing eddy current loss is established. The mathematical model of input impedance and signal transmission characteristics of the inductive coupler is established through circuit analysis. The influence of the electromagnetic parameters of the magnetic core and the load resistance of the coupler on the voltage transmission characteristics and power transmission characteristics of the coupler is studied. The research results show that the relative permeability of the magnetic core, load resistance of the coupler, and signal frequency seriously affect the signal voltage transmission. When the relative permeability of the magnetic core material reaches 50 , the voltage transmission of the inductive coupler is effectively improved. The electromagnetic simulation results are consistent with the theoretical analysis of the circuit model, which proves the correctness of the circuit model. This paper provides theoretical guidance for the electromagnetic design of inductive couplers and the improvement of signal transmission characteristics of telemetry drill string.
\end{abstract}

\section{Introduction}

Measurement while drilling/logging while drilling (MWD/ LWD) technology can transmit formation information to the surface in real time during drilling, but, limited by the transmission rate, the current formation sampling spacing is larger than that of conventional logging technology, which is not conducive to the discovery and exploitation of thin reservoirs [1]. A higher transmission rate of downhole information of MWD/LWD can make the drill bit travel along a predetermined track in real time to achieve safe drilling [2]. The telemetry drill string system $[3,4]$ uses a high-frequency electromagnetic signal to realize the high-speed wired transmission of downhole information, and the transmission rate can reach $2 \mathrm{Mbit} / \mathrm{s}$, which is much higher than the conventional wireless transmission technology of downhole information such as mud pressure pulse [5], formation electromagnetic wave [6], and drill string acoustic wave $[7,8]$, indicating good potential for practical application. The system is composed of a series of wired drill pipes. The structure of a wired drill pipe is shown in Figure 1.

In Figure 1, a coaxial cable is embedded in the rod body of a wired drill pipe, and an electrical signal is transmitted in the rod body. The spiral pin nose and box joint are located at each end of the rod body. The pin nose and box joint are grooved so that a ring-shaped high-frequency magnetic core 


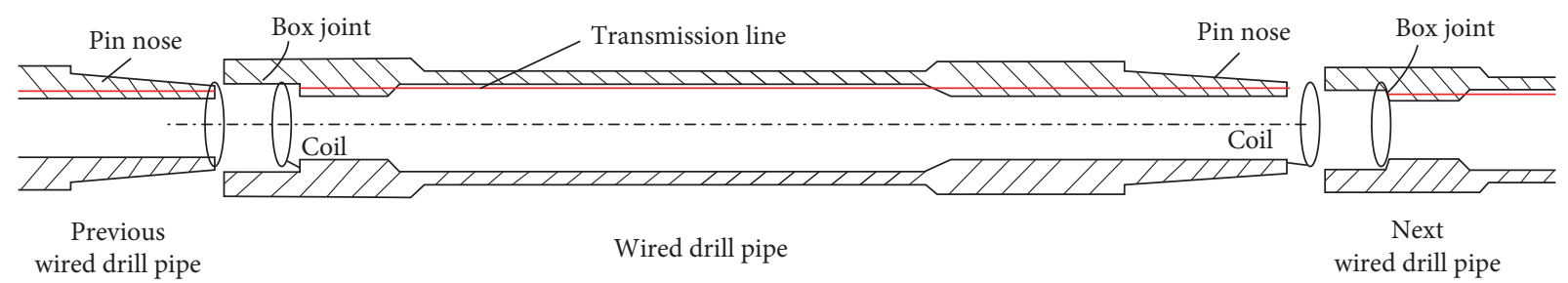

Figure 1: Cutaway of a wired drill pipe.

can be placed within them. The magnetic cores are also grooved, and the cable wires in the rod body are embedded in the magnetic core grooves at both ends of the drill pipe to form a single-turn coil. When two wired drill pipes are connected, the coil in the pin nose of the wired drill pipe and the coil in the box joint of the next wired joint form an inductive coupler, and the high-frequency signal is transmitted between the wired drill pipes through electromagnetic induction.

The voltage transfer coefficient of the inductor coupler has a great influence on the signal transmission of the telemetry drill string. How to improve the voltage transfer function value of the inductor coupler has become a key problem to be solved. Aiming at the improvement of the voltage transmission coefficient of the coupler, a few studies $[9,10]$ use an additional capacitor in parallel with the inductive coupler to form a resonant circuit to improve the signal transmission characteristics. Others [11-14] reduce the eddy current loss of the magnetic core by selecting lowconductivity magnetic materials or using special inserting technology. In [15], the coupling gap of the coupling coil is reduced by improving the surface roughness of the magnetic material to reduce the leakage of the magnetic field. Eddy current loss in the drill pipe is avoided in [16] by adding a copper gasket between the drill pipe joint groove and the magnetic core to shield the leakage magnetic field from entering the steel drill pipe. Several studies [17-19] analyze the transmission characteristics and optimize the performance of the coupler through circuit simulation, electromagnetic field simulation, and experimental methods. Some theoretical studies [20-22] are conducted on the characteristics of the coupler based on the equivalent high-frequency transformer model, but this model needs to be further improved to adapt to the research of inductive coupler in wired drill pipe.

The main problem with the above research is that it difficult to quantitatively describe the degree of influence and law of the relevant electromagnetic parameters on the voltage transfer function of the coupler. A method to solve this problem is through reasonable high-frequency circuit modeling and signal transmission mathematical analysis of the inductive coupler. The high-frequency circuit modeling of the inductive coupler needs to consider the influences of the eddy current loss of the magnetic core material, magnetic field leakage, and distributed capacitance of the coupler on signal transmission and ensure they are equivalent to the influences of the corresponding circuit elements. However, in the current coupler high-frequency transformer model, an equivalent resistance is connected in parallel to both ends of the primary coil to reflect the effect of eddy current loss. This equivalent method does not consider the interaction between eddy current and the primary and secondary coils of the coupler, so it cannot reflect the influence of eddy current on the current and voltage in the coupler circuit. Furthermore, it lacks strict physical meaning, which makes it difficult for the circuit analysis result to reflect the actual situation. Therefore, how to achieve a reasonable circuit equivalent is the key to circuit modeling.

This paper uses the electromagnetic detection and analysis method of nondestructive testing (NDT) to study the generation mechanism of eddy current in magnetic core material and the interaction mechanism between eddy current and the coupling coil; on this basis, a high-frequency circuit model of the coupler considering eddy current loss is established. The input impedance mathematical model and signal transmission characteristic mathematical model of the coupler are established through theoretical analysis. The relevant circuit parameters of the coupler are determined according to the calculation results of the mathematical model and the electromagnetic field finite element simulation analysis, and the voltage transmission characteristics and power transmission characteristics of the coupler are studied.

\section{Circuit Model and Circuit Analysis of Inductive Coupler}

2.1. Inductive Coupler Circuit Model. When wired drill pipes are connected in pairs, the coil embedded in the magnetic core groove at the pin nose of the wired drill pipe and the coil embedded in the magnetic core groove at the box joint of the adjacent drill pipe form a pair of coupling coils. Since the cross section of the magnetic core is coated with a protective layer, the two magnetic cores are not completely in contact, and there is a certain gap in the magnetic circuit of the coupling coils. The gap will cause magnetic field leakage and affect the coupling of the magnetic field between the coils. In order to avoid the transmission interference caused by the rapid change of the gap caused by the strong vibration of the drill string when the drill bit is drilling, the shock absorber is usually combined with the drill string vibration energy recovery method to reduce its impact [23].

When the coil is supplied with alternating current, the coupling coil forms a transformer. Due to the existence of the magnetic circuit gap, the magnetic field will leak to a certain extent. At high frequencies, the transformer coil has distributed capacitance and stray capacitance between coupling coils and eddy current loss of magnetic core. 
However, the stray capacitance is far less than the distributed capacitance. Since the signal frequency is around $10 \mathrm{MHz}$, the frequency is not very high, so the influence of the stray capacitance can be ignored. According to the electromagnetic detection and analysis method [24, 25], the eddy current generated in the magnetic core material will interact with the primary and secondary coils of the coupling coil to affect the coil current. Since the eddy current is affected by the material conductivity, this effect can be represented by the equivalent resistance of the eddy current ring. If the eddy current loop is equivalent to a closed single-turn coil, the eddy current loop has equivalent inductance and equivalent resistance. Considering the magnetic field leakage on the magnetic circuit from the primary to secondary coil, the high-frequency circuit model of the coupler can be constructed as shown in Figure 2.
In Figure 2, $r_{c}$ is the coil resistance; $L$ is the coil inductance; $L_{k}$ is the leakage inductance of coils; $M=L-L_{k}$ is the mutual inductance between coupling coils; $k=M / L$ is the coupling coefficient between coupling coils; $R_{L}$ is the load resistance of the secondary coil; $C_{p}$ is the distributed capacitance of the coil; $L_{e}$ is the equivalent inductance of the eddy current ring (due to the skin effect of high-frequency current, the eddy current is very close to the coil, and the equivalent radius of the eddy current ring is basically the same as that of the coil; thus, $\left.L_{e}=L\right) ; M_{e}$ is the mutual inductance between coil and eddy current ring (because the coil is fully wrapped by the magnetic core, the coil and eddy current ring are in a fully coupled state; thus, $M_{e}=L_{e}=L$ ); and $r_{e}$ is the equivalent resistance of the eddy current ring.

According to Figure 2, the circuit equations can be established as follows:

$$
\left\{\begin{array}{c}
\dot{U}_{1}=\left[r_{c}+j \omega\left(L-L_{k}\right)\right] \dot{I}_{1}+j \omega L_{k} \dot{I}_{1}+j \omega M \dot{I}_{3}+j \omega M_{e} \dot{I}_{2}, \\
\left(r_{e}+j \omega L_{e}\right) \dot{I}_{2}+j \omega M_{e}\left(\dot{I}_{1}-\dot{I}_{3}\right)=0, \\
R_{L} \dot{I}_{5}-j \omega M_{e} \dot{I}_{2}+\left[r_{c}+j \omega\left(L-L_{k}\right)\right] \dot{I}_{3}+j \omega L_{k} \dot{I}_{3}+j \omega M \dot{I}_{1}=0, \\
R_{L} \dot{I}_{5}=\frac{\dot{I}_{4}}{j \omega C_{p}}, \\
\dot{I}_{3}=\dot{I}_{4}+\dot{I}_{5},
\end{array}\right\}
$$

where $j \omega M \dot{I}_{1}$ and $j \omega M \dot{I}_{3}$ are mutually induced electromotive forces generated between the coupled coils; $j \omega M_{e} \dot{I}_{2}$ is the mutually induced electromotive force generated by the eddy current loop in the coupling coil; $j \omega M_{e}\left(\dot{I}_{1}-\dot{I}_{3}\right)$ is the mutually induced electromotive force generated by the coupling coil in the eddy current loop; $\dot{U}$ is the voltage phasor; $\dot{I}$ is the current phasor; $\omega=2 \pi f$ is the angular frequency; $f$ is the signal frequency; and $j$ represents the imaginary part of a complex number.

\subsection{Circuit Analysis}

2.2.1. Input Impedance of Inductive Coupler. The input impedance of the coupler reflects the influence of the current in secondary coil and the eddy current in the magnetic core on the primary coil current. According to equation (1), through mathematical derivation, the input impedance of the circuit viewed from both ends of the primary coil can be obtained as

$$
Z_{\text {in }}=\frac{\dot{U}_{1}}{\dot{I}_{1}}=R_{\text {in }}+j X_{\text {in }}
$$

where 


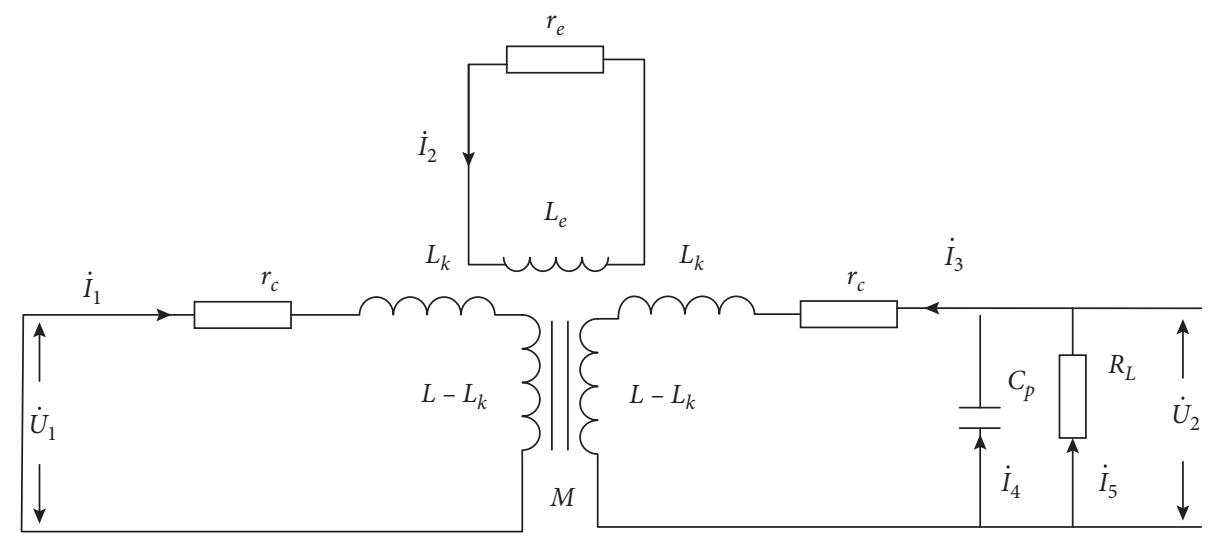

FIGURE 2: High-frequency circuit model of inductive coupler.

In equation (2), $\left\{r_{f 1}=\left(\omega^{2} M_{e_{3}}^{2} r_{e} / r_{e}^{2}+\omega^{2} L_{e}^{2}\right)+([\omega L\right.$ $\left.-\omega C_{p} R_{L}^{2}\left(1-\omega^{2} L C_{p}\right)\right]\left(2 \omega^{3} M^{2} L_{e}+2 \omega^{3} M_{e}^{2} M\right) / r_{e} R_{L}^{2}\left(1-\omega^{2}\right.$ $\left.\left.L C_{p}\right)^{2}+r_{e} \omega^{2} L^{2}\right) X_{f 1}=-\left(\omega^{3} M_{e}^{2} L_{e} / r_{e}^{2}+\omega^{2} L_{e}^{2}\right)+\left(R_{L}\left[2 \omega^{3} M^{2}\right.\right.$ $\left.\left.\left.L_{e}+2 \omega^{3} M_{e}^{2} M\right] / r_{e} R_{L}^{2}\left(1-\omega^{2} L C_{p}\right)^{2}+r_{e} \omega^{2} L^{2}\right)\right\}$ is the influence of the eddy current on the input impedance of the coupler.
2.2.2. Voltage Transfer Function. The voltage transfer function of the inductive coupler is the ratio of the load voltage phasor modulus to the input voltage phasor modulus, which reflects the voltage transmission capability of the coupler. According to equation (1), through mathematical derivation, the mathematical model of the voltage transfer function can be obtained as follows:

$$
\eta_{U}=\left|\frac{\dot{U}_{2}}{\dot{U}_{1}}\right|=\frac{R_{L} \sqrt{\omega^{4} L_{e}^{2}\left(2 L-L_{k}\right)^{2}+\omega^{2}\left(L-L_{k}\right)^{2} r_{e}^{2}}}{\sqrt{\left[\omega^{2} L_{k} r_{e}\left(2 L-L_{k}\right)+\omega^{4} R_{L} C_{p} L_{e}\left(2 L-L_{k}\right)^{2}\right]^{2}+\left[\omega L r_{e} R_{L}+\omega^{3} L_{e}\left(2 L-L_{k}\right)^{2}-\omega^{3} C_{p} R_{L} r_{e} L_{k}\left(2 L-L_{k}\right)\right]^{2}}} .
$$

In equation (4), under the following extreme conditions: the distributed capacitance $C_{p}=0$; the load resistance $R_{L} \longrightarrow \infty$; and the equivalent resistance of eddy current ring $r_{e} \longrightarrow \infty$ or the magnetic core conductivity $\sigma=0$, we can get the voltage transfer function $\eta_{U} l_{\text {ext }}=\left(L-L_{k} / L\right)=k$. This means that the coupling coefficient can be obtained by the electromagnetic simulation of $\left.\eta_{U}\right|_{\text {ext }}$.

2.2.3. Power Transfer Function. The power transfer function of the inductive coupler is the ratio of the active power obtained on the load resistance to the input active power, which reflects the capability of the load to obtain active power from the input of the coupler. The electric power of the coupler is composed of the active power consumed by resistance and the reactive power stored in the reactance element. Because the load of the coupler is a pure resistive element, the electric power on the load is the power consumed by the load resistance. Through energy analysis, the active power consumed by the coupler at high-frequency includes the power consumed by the coil resistance, the eddy current loss inside the magnetic core, and the power consumed by the load, which can also be expressed as the real part of the input power of the coupler. According to the input impedance analysis of the coupler, the electrical power of the coupler can be described as

$$
P_{\text {in }}=\frac{\left|\dot{U}_{1}\right|^{2}}{Z_{\text {in }}}=\frac{\left|\dot{U}_{1}\right|^{2}}{R_{\text {in }}+j X_{\text {in }}}=\frac{\left|\dot{U}_{1}\right|^{2}\left(R_{\mathrm{in}}-j X_{\text {in }}\right)}{R_{\text {in }}^{2}+X_{\text {in }}^{2}}=\frac{\left|\dot{U}_{1}\right|^{2} R_{\text {in }}}{R_{\text {in }}^{2}+X_{\text {in }}^{2}}+j \frac{-\left|\dot{U}_{1}\right|^{2} X_{\text {in }}}{R_{\text {in }}^{2}+X_{\text {in }}^{2}}=R_{e}\left[P_{\text {in }}\right]+I_{m}\left[P_{\text {in }}\right]
$$

where $R_{e}\left[P_{\text {in }}\right]=\left(\left|\dot{U}_{1}\right|^{2} R_{\text {in }} / R_{\text {in }}^{2}+X_{\text {in }}^{2}\right)$ is active power.

According to equation (2), the mathematical model of the active power of the inductive coupler can be established as 


$$
R_{e}\left[P_{\mathrm{in}}\right]=\frac{\left|\dot{U}_{1}\right|^{2}}{R_{\mathrm{in}}^{2}+X_{\mathrm{in}}^{2}}\left\{r_{c}+\frac{\omega^{2} M_{e}^{2} r_{e}}{r_{e}^{2}+\omega^{2} L_{e}^{2}}+\frac{\omega^{2} M^{2} R_{L}}{R_{L}^{2}\left(1-\omega^{2} L C_{p}\right)^{2}+\omega^{2} L^{2}}+\frac{\left[\omega L-\omega C_{p} R_{L}^{2}\left(1-\omega^{2} L C_{p}\right)\right]\left(2 \omega^{3} M^{2} L_{e}+2 \omega^{3} M_{e}^{2} M\right)}{r_{e} R_{L}^{2}\left(1-\omega^{2} L C_{p}\right)^{2}+r_{e} \omega^{2} L^{2}}\right\} .
$$

In equation (6), $P_{c}=\left(\left|\dot{U}_{1}\right|^{2} r_{c} / R_{\mathrm{in}}^{2}+X_{\mathrm{in}}^{2}\right)$ is the power of coil resistance; $\quad P_{L}=\left(\left|\dot{U}_{1}\right|^{2} \omega^{2} M^{2} R_{L} /\left(R_{\mathrm{in}}^{2}+X_{\mathrm{in}}^{2}\right)\left[R_{L}^{2}(1\right.\right.$ $\left.\left.\left.-\omega^{2} L C_{p}\right)^{2}+\omega^{2} L^{2}\right]\right)$ is the power of load; $\left(\left|\dot{U}_{1}\right|^{2} \omega^{2} M_{e}^{2} r_{e} /\left(R_{\text {in }}^{2}\right.\right.$ $\left.\left.+X_{\text {in }}^{2}\right)\left(r_{e}^{2}+\omega^{2} L_{e}^{2}\right)\right)$ is the power directly consumed by the eddy current; and $\left(\left|\dot{U}_{1}\right|^{2}\left[\omega L-\omega C_{p} R_{L}^{2}\left(1-\omega^{2} L C_{p}\right)\right]\right.$ $\left(2 \omega^{3} M^{2} L_{e}+2 \omega^{3} M_{e}^{2} M\right) /\left(R_{\mathrm{in}}^{2}+X_{\mathrm{in}}^{2}\right)\left[r_{e} R_{L}^{2}\left(1-\omega^{2} L C_{p}\right)^{2}+\right.$ $\left.\left.r_{e} \omega^{2} L\right]\right)$ is the power caused by $r_{e}$ and $R_{L}$. For further analysis of this term, if $r_{e} \longrightarrow \infty$, then this term is equal to zero regardless of the value of $R_{L}$. This indicates that this term is mainly indirectly affected by $r_{e}$. Therefore, the term can be classified as the power consumed by eddy current.

Thus, the active power transfer function of the inductive coupler can be expressed as

$$
\eta_{P}=\frac{P_{L}}{R_{e}\left[P_{\text {in }}\right]}=\frac{\omega^{2} M^{2} R_{L} r_{e}}{\left(r_{c} r_{e}+\omega^{2} L_{e}^{2}\right)\left[R_{L}^{2}\left(1-\omega^{2} L C_{p}\right)^{2}+\omega^{2} L^{2}\right]+\omega^{2} M^{2} R_{L} r_{e}+2 \omega^{3} L_{e} M\left(M+M_{e}\right)\left[\omega L-\omega C_{p} R_{L}^{2}\left(1-\omega^{2} L C_{p}\right)\right]}
$$

\section{Numerical Calculation and Simulation Analysis}

\subsection{Calculation Parameters}

3.1.1. Electromagnetic Simulation Model and Parameters of Inductive Coupler. The electromagnetic simulation is performed using COMSOL 5.4 software, the magnetic core is made of $\mathrm{Ni}-\mathrm{Zn}$ ferrite, the coil conductor is made of copper, and the structural model of the coupler is shown in Figure 3, where $d_{1}$ is the inner diameter of the core; $d_{2}$ is the outer diameter of the core; $H$ is the height of the core; $D_{e}$ is the depth of the groove; $W$ is the width of the groove; $g$ is the gap of the magnetic circuit; $d_{c}$ is the coil diameter; and $d_{0}$ is the outer diameter of the coil conductor.

The conductivity of Ni-Zn ferrite usually used in highfrequency band is less than $1 \mathrm{~S} / \mathrm{m}$, and the relative permeability can reach several hundred. Because the relative permeability of the material is greatly affected by temperature, the higher the relative permeability and the bigger the temperature coefficient. The working temperature of the wired drill pipe is close to $100^{\circ} \mathrm{C}$. In order to reduce the influence of temperature on the relative permeability of the magnetic core, value of the relative permeability is usually selected at about 10 .

The magnetic core structural parameters and electromagnetic parameters are as follows: $d_{1}=123 \mathrm{~mm}$; $d_{2}=147 \mathrm{~mm} ; \quad H=8 \mathrm{~mm} ; \quad D_{e}=4 \mathrm{~mm} ; W=2 \mathrm{~mm} ;$ $g=0.5 \mathrm{~mm}$; relative permeability $\mu_{r}=10$; and conductivity $\sigma=1 \mathrm{~S} / \mathrm{m}$. The coil is $1 \mathrm{turn}$; the coil diameter $d_{c}=134 \mathrm{~mm}$; the outer diameter of the coil conductor $d_{0}=1 \mathrm{~mm}$; the conductor resistivity $\rho_{c}=1.67 \times 10^{-8} \Omega \cdot \mathrm{m}$; and coil resistance $r_{c}=9 \times 10^{-3} \Omega$. The distributed capacitance $C_{p}=50 \mathrm{pF}$; the load resistance $R_{L}=75 \Omega$; the voltage of the signal source $\dot{U}_{1}=1 \mathrm{~V}$; and the signal frequency varies in range of $f=0.5-20 \mathrm{MHz}$.
3.1.2. Determination of Parameters Using Combination of Electromagnetic Simulation and Mathematical Analysis. The numerical calculation of inductive coupler signal transmission requires electromagnetic parameters, such as coil inductance, leakage inductance, coupling coefficient, and equivalent resistance of eddy current ring, to be defined. These parameters can be determined by electromagnetic simulation combined with mathematical analysis. Because the coupler transmits a frequency band signal with a certain bandwidth, the signal contains many frequency components, so it is necessary to understand the variation rule of the above parameters with respect to frequency.

(1) Coil Inductance of Inductive Coupler. Under the conditions of $C_{p}=0, R_{L} \longrightarrow \infty, \sigma=0$, and $\mu_{r}=10$, the curve of coil inductance plotted against signal frequency can be obtained by electromagnetic simulation, as shown in Figure 4.

It can be seen from Figure 4 that the coil inductance $L=1.234 \mu \mathrm{H}$, and it is independent of frequency. This indicates that the coil inductance is constant when the magnetic core structural and electromagnetic parameters are determined.

(2) Coupling Coefficient of Inductive Coupler. Under the conditions of $C_{p}=0, R_{L} \longrightarrow \infty, \sigma=0$, and $\mu_{r}=10$, according to $\left.\eta_{U}\right|_{\text {ext }}=\left(L-L_{k} / L\right)=k$, the curve of coupling coefficient of inductive couplers versus signal frequency can be obtained by electromagnetic simulation, as shown in Figure 5 .

It can be seen from Figure 5 that the coupling efficient remains a constant of $k=0.468$. The leakage inductance can be calculated from the coupling coefficient and coil inductance by $L_{k}=L(1-k)=0.532 \mu \mathrm{H}$.

(3) Equivalent Resistance of Eddy Current Ring. Under the conditions of $C_{p}=50 \mathrm{pF}, R_{L}=75 \Omega, \sigma=1 \mathrm{~S} / \mathrm{m}$, and $\mu_{r}=10$, the input resistance $R_{\mathrm{in}}$ is obtained from electromagnetic simulation as follows: $R_{\mathrm{in}}=r_{c}+$ $\left(\omega^{2} M_{e}^{2} r_{e} / r_{e}^{2}+\omega^{2} h_{e}^{2}\right)$ 


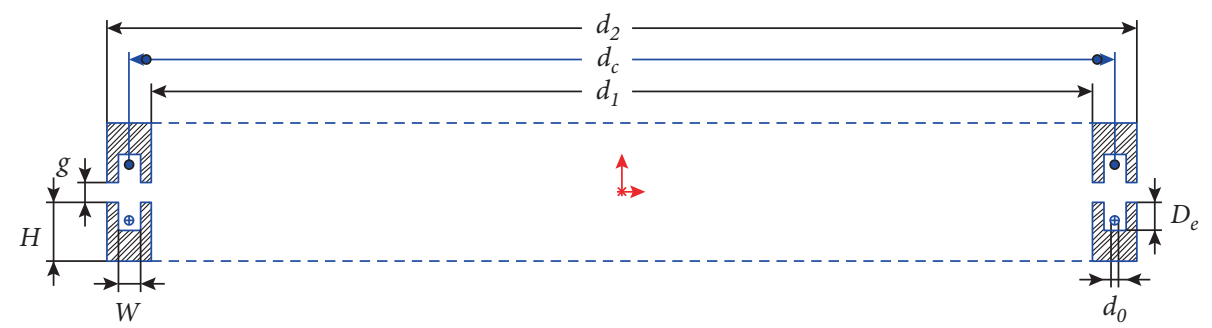

FIGURE 3: Electromagnetic simulation model of inductive coupler.

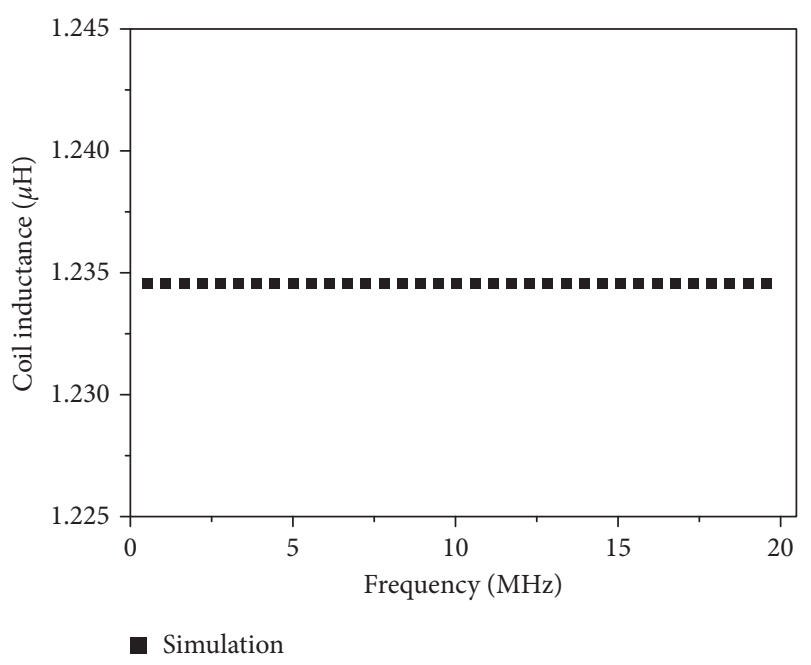

FIGURE 4: Influence of signal frequency on coil inductance.

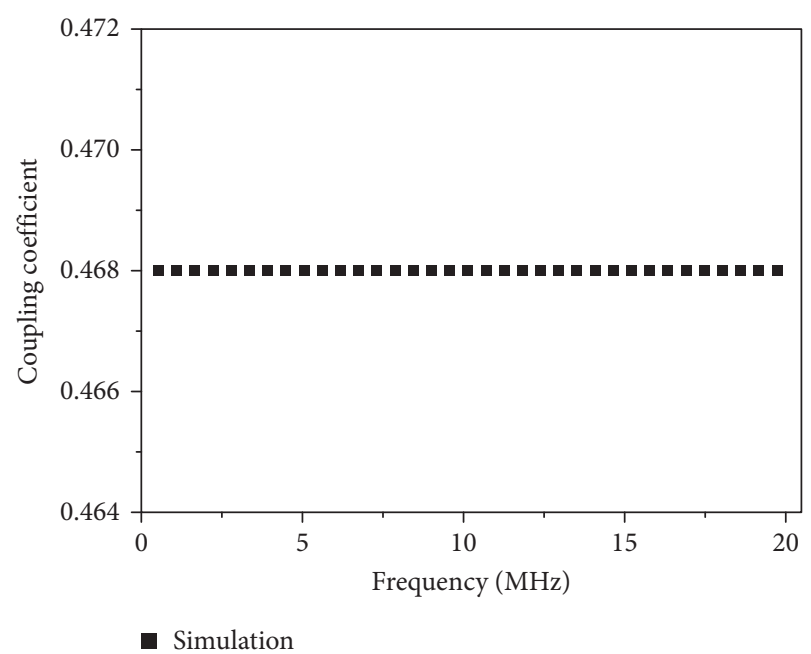

Figure 5: Influence of signal frequency on coupling coefficient.

$+\left(\left[\omega L-\omega C_{p} R_{L}^{2}\left(1-\omega^{2} L C_{p}\right)\right]\left(2 \omega^{3} M^{2} L_{e}+2 \omega^{3} M_{e}^{2} M\right) / r_{e} R_{L}^{2}\right.$ $\left.\left(1-\omega^{2} L C_{p}\right)^{2}+r_{e} \omega^{2} L^{2}\right)+\left(\omega^{2} M^{2} R_{L} / R_{L}^{2}\left(1-\omega^{2} L C_{p}\right)^{2}\right.$ $\left.+\omega^{2} L^{2}\right)$. The equivalent resistance of eddy current ring at different frequencies is obtained by inversing $R_{\text {in }}$ with MATLAB, and the curve is shown in Figure 6.

It can be seen from Figure 6 that the equivalent resistance of eddy current ring monotonously increases with

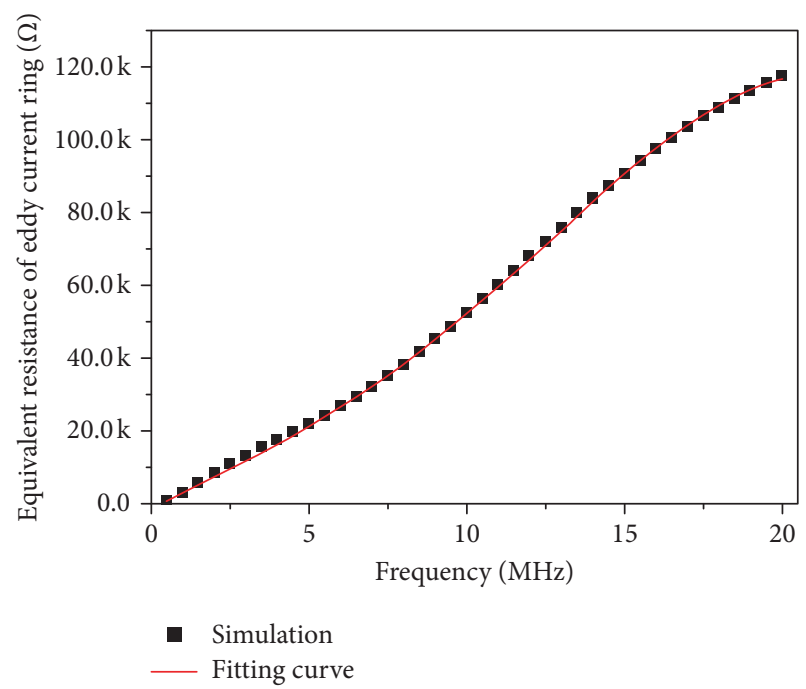

FIGURE 6: Influence of signal frequency on equivalent resistance of eddy current ring.

frequency, and the variation law with respect to frequency can be obtained by numerical fitting as $r_{e}=-1400+4.75 \times$ $10^{-3} f-1.96 \times 10^{-10} f^{2}+3.85 \times 10^{-17} f^{3}-1.29 \times 10^{-24} f^{4}$.

\subsection{Influence of Electromagnetic Parameters of Magnetic Core Material on Signal Transmission}

3.2.1. Influence of Relative Permeability on Transfer Function of the Coupler. Under the conditions of $C_{p}=50 \mathrm{pF}$, $R_{L}=75 \Omega, \sigma=1 \mathrm{~S} / \mathrm{m}$, and $f=10 \mathrm{MHz}$, the relative permeability varies in the range of $\mu_{r}=10-200$. The variation curves of coil inductance, coupling coefficient, and equivalent resistance of eddy current ring with respect to the relative permeability of the magnetic core are shown in Figure 7.

It can be seen from Figure 7 that the inductance and coupling coefficient of the inductive coupler are greatly affected by the relative permeability of the magnetic core and monotonously increase with the increase in relative permeability. A higher coupling coefficient can be obtained in a larger relative permeability, which is beneficial for signal transmission. Through numerical fitting, the functional relationship between coil inductance and relative permeability is $L=0.73+5.9 \times 10^{-8} \mu_{r}-5.99 \times 10^{-10} \mu_{r}^{2}+2.94 \times 10^{-12} \mu_{r}^{3}$ $-5.45 \times 10^{-15} \mu_{r}^{4}$, and the functional relationship between coupling coefficient and relative permeability is $k=0.37+$ 


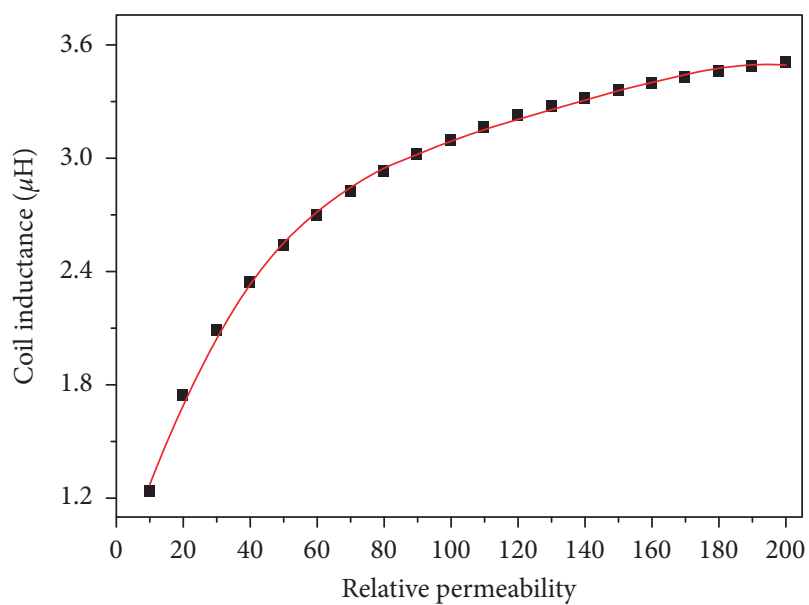

- Simulation Fitting curve

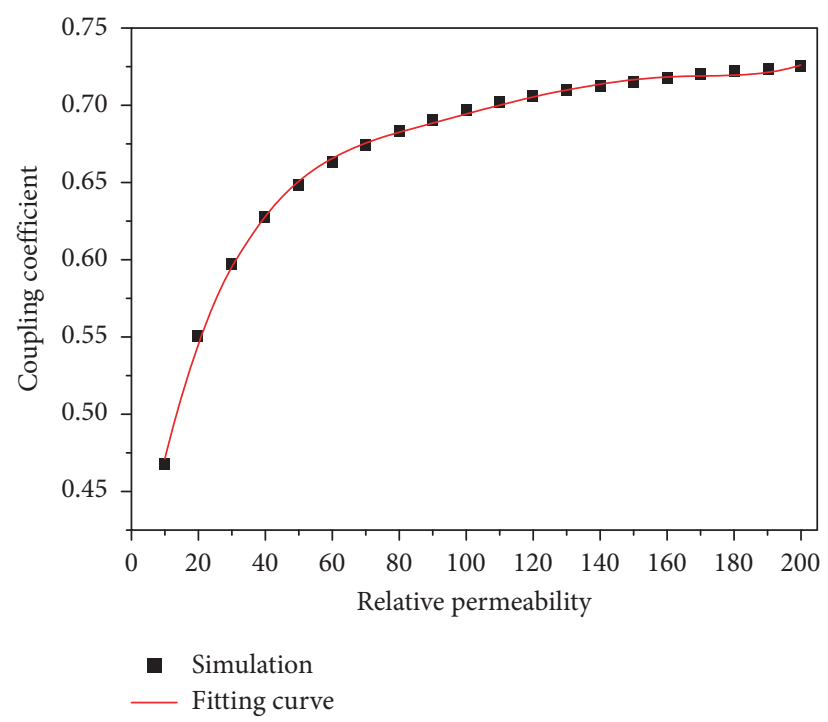

(b)

(a)

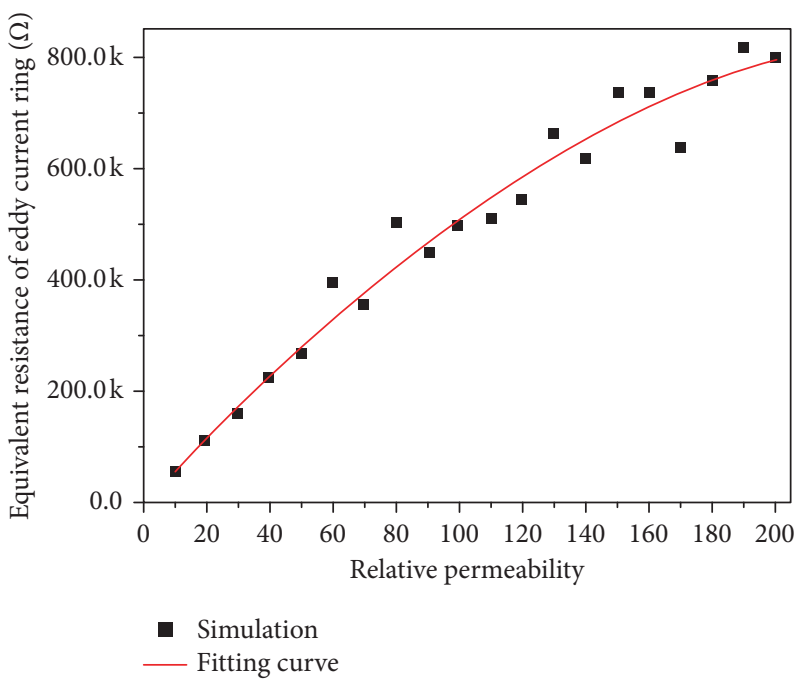

(c)

FiguRE 7: Influence of core relative permeability on electromagnetic parameters of the coupler: (a) coil inductance of the coupler; (b) coupling coefficient; (c) equivalent resistance of eddy current ring.

$0.0124 \mu_{r}-2.04 \times 10^{-4} \mu_{r}^{2}+1.73 \times 10^{-6} \mu_{r}^{3} \quad-7.18 \times 10^{-9} \mu_{r}^{4}$ $+1.15 \times 10^{-11} \mu_{r}^{5}$. The equivalent resistance of eddy current ring increases monotonously with the change of relative permeability, but the value fluctuates greatly. The equivalent resistance of the eddy current ring as a function of the relative permeability is obtained by numerical fitting as $r_{e}=-5796.39+6320 \mu_{r}-11.54 \mu_{r}^{2}$. Figure 8 shows the simulated results and the calculated results of the signal transfer function value of the coupler with the relative permeability obtained using the mathematical model including equations (4) and (7) under the condition of $r_{e}=-5796.39+6320 \mu_{r}-11.54 \mu_{r}^{2}$.

The following can be seen from Figure 8. (1) The increasing permeability can improve the voltage transfer function value of the inductive coupler to a certain extent, but the influence of relative permeability on the voltage transfer function gradually becomes smaller when the relative permeability is greater than 50 . Thus, when selecting the core material, the relative permeability of 50 is more appropriate. (2) The relative permeability has little influence on the power transfer function. The power transfer function value remains above $95 \%$, which indicates that the eddy current loss has little effect.

\subsubsection{Influence of Core Conductivity on Transfer Function of} the Coupler. According to electromagnetic theory, the coil inductance is only related to the structure of the coil and the permeability of the magnetic core material and has nothing to do with the conductivity of the magnetic core; in the same way, the coupling coefficient reflects the coupling degree of magnetic field between coils and is also related to the relative permeability of the core material but still has nothing to do with the conductivity of the core. Therefore, the core 


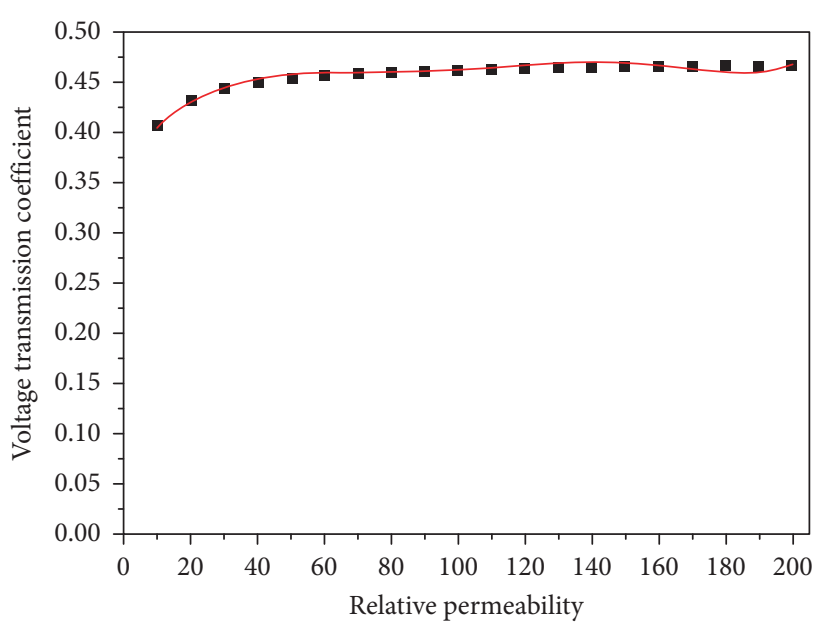

Simulation

Calculation

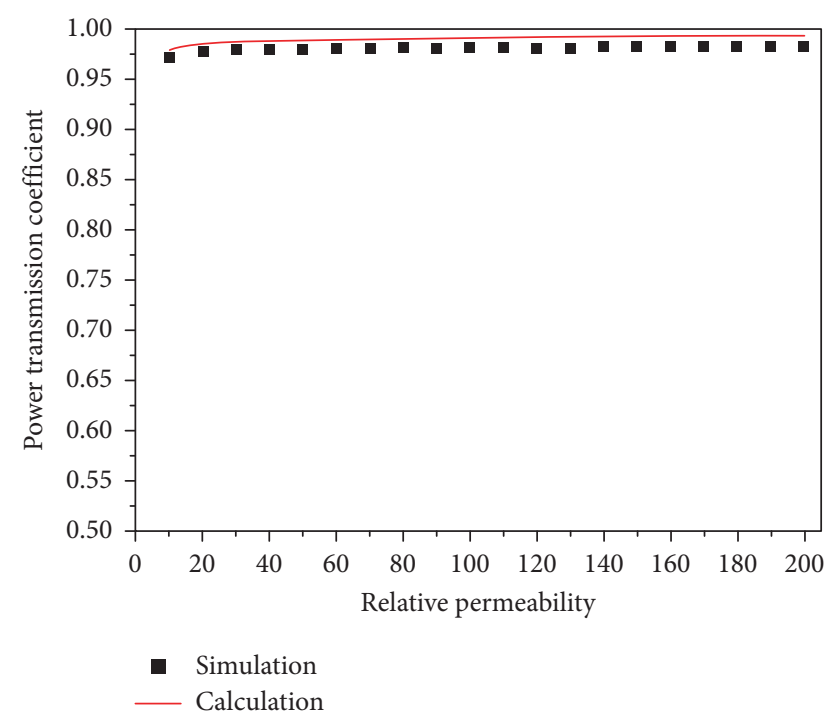

(b)

Figure 8: Influence of core relative permeability on transfer function of the coupler: (a) voltage transfer function; (b) power transfer function.

conductivity will not affect the inductance and coupling coefficient of the coupler but will only affect the equivalent resistance of the eddy current ring.

Under the conditions of $C_{p}=50 \mathrm{pF}, R_{L}=75 \Omega$, $f=10 \mathrm{MHz}$, and $\mu_{r}=10$, the variation range of core conductivity is in the range of $\sigma=0.2-2 \mathrm{~S} / \mathrm{m}$. The equivalent resistance of the eddy current ring varies with the conductivity of magnetic core as shown in Figure 9.

It can be seen from Figure 9 that as the core conductivity increases, the equivalent resistance of eddy current ring decreases. Though numerical fitting, the functional relationship between the equivalent resistance of eddy current ring and the core conductivity is $r_{e}=3.03 \times 10^{5}-7.14 \times$ $10^{5} \sigma+7.83 \times 10^{5} \sigma^{2}-3.91 \times 10^{5} \sigma^{3}+7.21 \times 10^{4} \sigma^{4}$. Then, the calculated results of coupler voltage and power transfer function value according to equations (4) and (7) with different core conductivity and simulated results are shown in Figure 10.

It can be seen from Figure 10 that the calculated values of the voltage transfer function and power transfer function are basically consistent with the simulation analysis results. With the increase in core conductivity, the values of voltage transfer function and power transfer function decrease, but the change is small, indicating that the core conductivity has little effect on the signal transmission of the inductive coupler.

3.3. Analysis of Signal Transmission Characteristics of Inductive Coupler. The signal transmission characteristic of the coupler is the influence of frequency on the transmission function, including the voltage transmission characteristic and the power transmission characteristic, which reflects the transmission capability of the coupler for different frequency signals. Under the conditions of $\mu_{r}=10, \sigma=1 \mathrm{~S} / \mathrm{m}$, $C_{p}=50 \mathrm{pF}, \quad R_{L}=75 \Omega, \quad L=1.234 \mu \mathrm{H}, \quad k=0.468$,

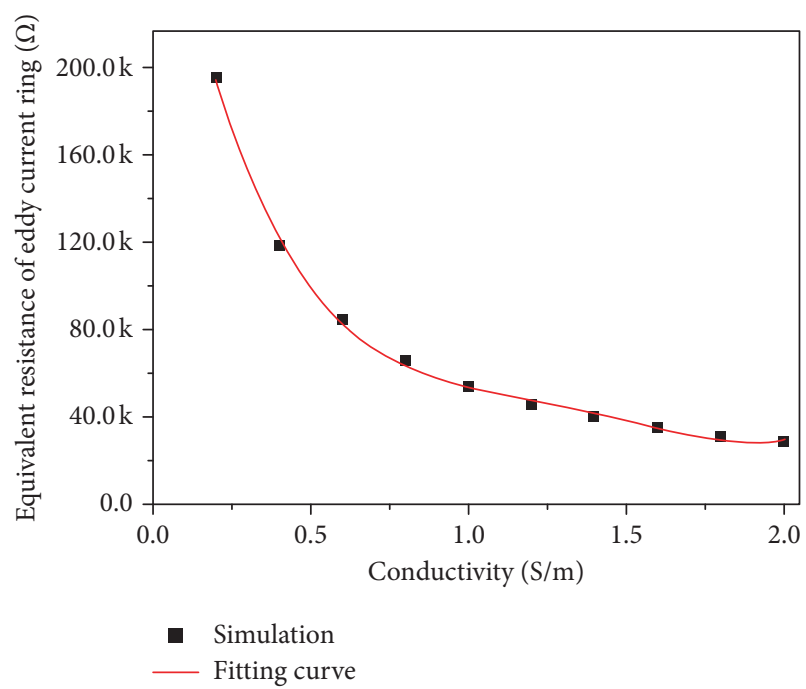

FIGURE 9: Influence of core conductivity on equivalent resistance of eddy current ring.

$L_{k}=L(1-k)=0.656 \mu \mathrm{H}, \quad M=L-L_{k}=0.532 \mu \mathrm{H}, \quad$ and $r_{e}=-1400+4.75 \times 10^{-3} f-1.96 \times 10^{-10} f^{2}+3.85 \times$

$10^{-17} f^{3}-1.29 \times 10^{-24} f^{4}$, the simulated signal transmission characteristics and calculated signal transmission characteristics with respect to frequency, obtained using equations (4) and (7), are shown in Figure 11.

The four following observations can be made based on Figure 11. First, the calculated values of voltage transmission characteristics and power transmission characteristics are consistent with the simulation analysis results, which indicates the correctness of the established mathematical models of voltage transmission function and power transmission function of the coupler; after determining the relevant circuit parameters, the 


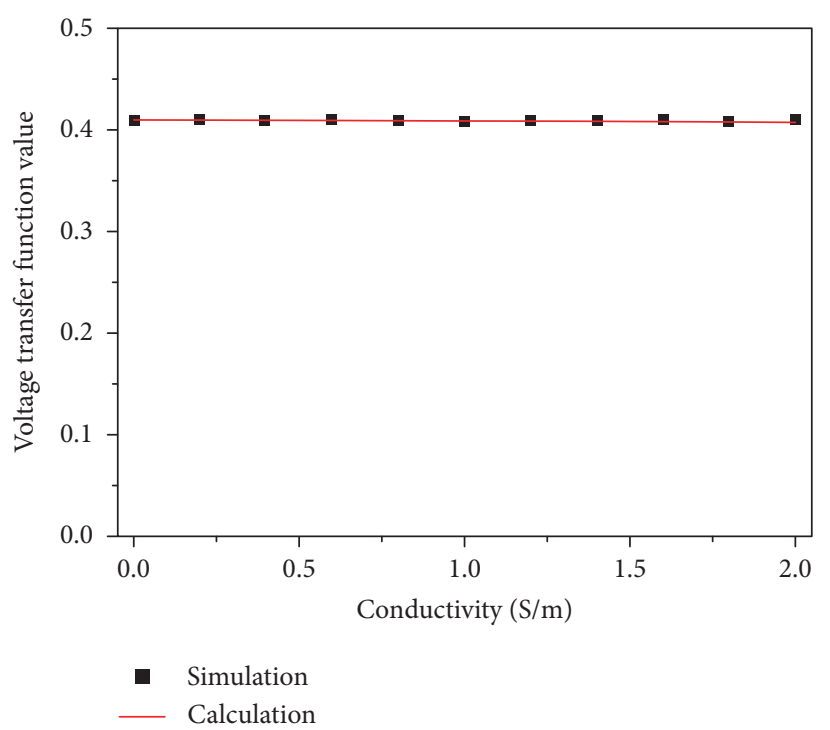

(a)

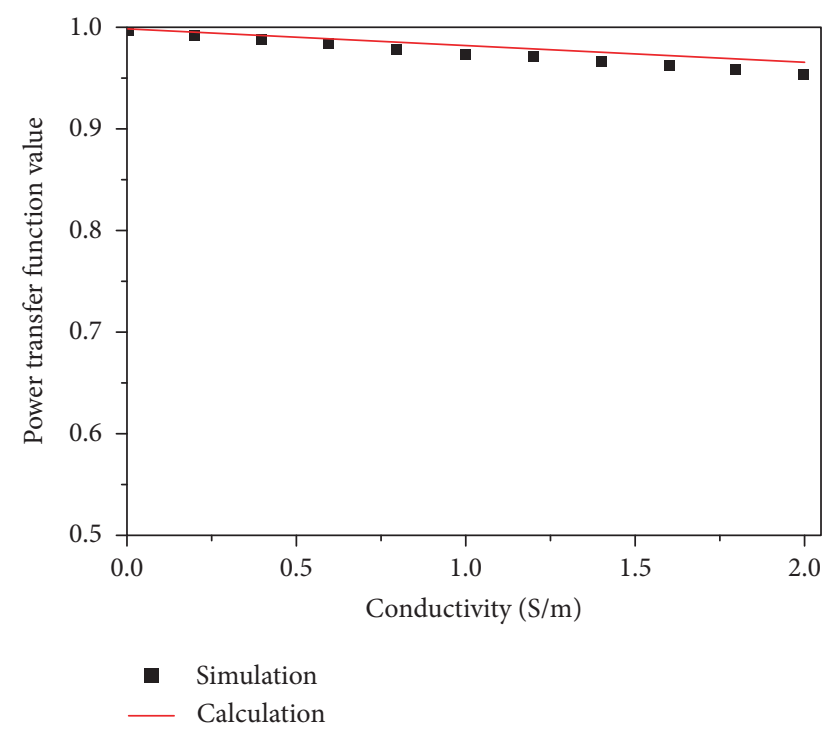

(b)

FIGURE 10: Influence of core conductivity on transfer function of coupler: (a) voltage transfer function; (b) power transfer function.

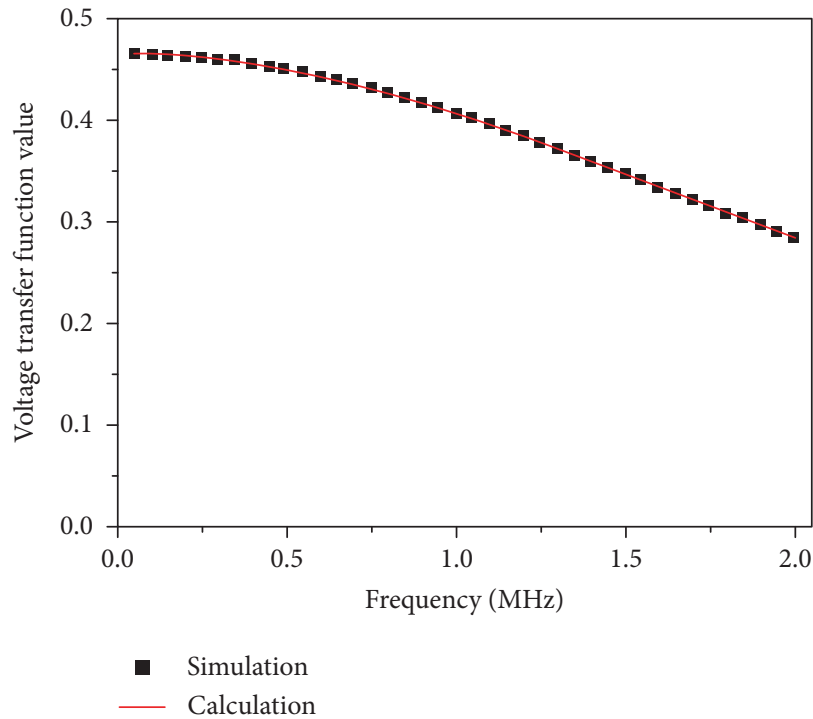

(a)

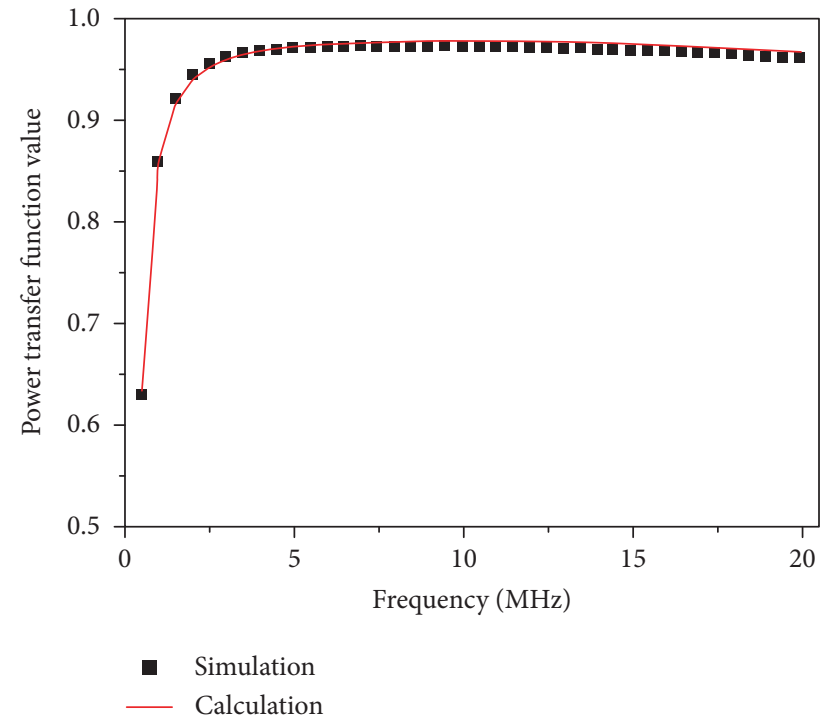

(b)

FiguRE 11: Signal transmission characteristics of inductive coupler: (a) voltage transmission characteristics; (b) power transmission characteristics.

quantitative calculation and analysis of the coupler signal transmission characteristics can be realized through the mathematical models of equations (4) and (7). Second, frequency has a great influence on the voltage transfer function of the inductive coupler. When the frequency is higher than $3 \mathrm{MHz}$, the voltage transfer function of the coupler decreases rapidly with the increase in frequency. The voltage transfer characteristic presents obvious lowpass characteristics, which are unfavorable to high-frequency signal transmission. Because the signal frequency is higher than $4 \mathrm{MHz}$, the voltage transmission characteristic of the coupler cannot realize effective signal transmission, so a relevant solution must be implemented to compensate for the transmission characteristics of the telemetry drill string channel to meet the transmission requirements of the frequency band signal [26]. Third, compared with the voltage transmission function of the coupler when the secondary coil is opening as shown in Figure 4, the voltage transmission function value of the coupler does not change with frequency. The load generates current at the secondary coil, the current is affected by frequency, and the change 
of current causes the output voltage of the coupler to change with frequency. The calculated results show that the smaller the load resistance, the greater the influence of frequency on the voltage transfer function of the coupler. Fourth, the frequency has a great influence on the power transfer function of the coupler, but when the frequency is higher than $3 \mathrm{MHz}$, the power transfer function values of the couplers are higher than 95\%, which indicates that the eddy current loss has little influence on the power transmission, and the load can obtain most of the active input power. It also indicates that the magnetic core will not produce large signal energy loss when the frequency is higher than $3 \mathrm{MHz}$, which is conducive to the long-distance transmission of the telemetry drill string signal.

\section{Conclusion}

The modeling of the high-frequency circuit of the inductive coupler needs to consider the influence of the eddy current loss of the magnetic core material, magnetic field leakage, and distributed capacitance on the signal transmission and is equivalent to the influence of the corresponding circuit elements. The electromagnetic detection and analysis method based on NDT is used to study the generation mechanism of eddy current in the magnetic core material and the interaction mechanism between eddy current and coupling coils. Circuit modeling with eddy current ring equivalent inductance and eddy current ring equivalent resistance can better reflect the influence of eddy current loss on signal transmission.

According to the mathematical model of the input impedance of the primary coil of the inductive coupler and the mathematical model of the voltage transfer function under extreme conditions, combined with the electromagnetic field simulation analysis to determine the relevant circuit parameters of the coupler, the quantitative calculation and analysis of the signal transmission characteristics of the coupler can be realized. The calculation results are consistent with the electromagnetic field simulation analysis results.

The magnetic permeability of the core material, load resistance, and signal frequency seriously affect the transmission of voltage signals, and the conductivity of the core material has a small effect on signal transmission. When there is a certain gap between the coupling coils, the relative permeability of the core can reach 50 , which effectively improves the voltage transmission of the coupler.

\section{Data Availability}

The data used in this article are given as figures.

\section{Conflicts of Interest}

The authors declare that they have no conflicts of interest.

\section{Authors' Contributions}

All authors approved the manuscript for publication.

\section{Acknowledgments}

This work was financially supported by the Fundamental Research Funds for the Central Universities of China (no. 27R1710013A) and the High-tech Research and Development Program of China (no. 2006AA06A101). The authors thank LetPub (http://www.letpub.com) for its linguistic assistance during the preparation of this manuscript.

\section{References}

[1] J. Vij, R. Saraiya, S. Saumya et al., "LWD as the absolute formation evaluation technology: present-day capabilities, limitations, and future developments of LWD technology," in Proceedings of the SPWLA 2nd Asia Pacific Technical Symposium, p. 15, Bogor, Java, Indonesia, November 2018.

[2] M. Giltner, L. Earle, J. Willis et al., "Performance impact of downhole data from wired drill pipe and downhole sensors," in Proceedings of the SPE/IADC International Drilling Conference and Exhibition 2019, Society of Petroleum Engineers, The Hague, The Netherlands, March 2019.

[3] F. Poletto, F. Miranda, P. Corubolo, A. Schleifer, and P. Comelli, "Drill-bit seismic monitoring while drilling by downhole wired-pipe telemetry," Geophysical Prospecting, vol. 62, no. 4, pp. 702-718, 2014.

[4] R. A. Shishavan, C. Hubbell, H. Perez, J. Hedengren, and D. Pixton, "Combined rate of penetration and pressure regulation for drilling optimization by use of high-speed telemetry," SPE Drilling \& Completion, vol. 30, no. 01, pp. 17-26, 2015.

[5] M. J. Berro and M. Reich, "Laboratory investigations of a hybrid mud pulse telemetry (HMPT) A new approach for speeding up the transmitting of MWD/LWD data in deep boreholes," Journal of Petroleum Science and Engineering, vol. 183, 2019.

[6] J. Schnitger and J. Macpherson, "Signal attenuation for electromagnetic telemetry systems," in Proceedings of the SPE/ IADC Drilling Conference and Exhibition, Society of Petroleum Engineers, Amsterdam, The Netherlands, March 2009.

[7] C. Li, J. Chang, S. Fan et al., "Analyzing the validity of a DFTbased improved acoustic OFDM transmission along rotating simulated drillstring," Mechanical Systems and Signal Processing, vol. 81, pp. 447-460, 2016.

[8] D. Ma, Y. Shi, W. Zhang, and G. Liu, "Design of acoustic transmission along drill strings for logging while drilling data based on adaptive NC-OFDM," AEU - International Journal of Electronics and Communications, vol. 83, pp. 329-338, 2018.

[9] M. Briscoe, J. McPherson, D. S. Pixton et al., "Filter for a drill string," U. S. Patent: 7303029 B2, 2007.

[10] D. R. Hall, D. S. Pixton, M. L. Johnson et al., "Downhole transmission system comprising a coaxial capacitor," U. S. Patent: 7948395 B2, 2011.

[11] T. Hall, C. Sneddon, D. S. Pixton et al., "Element for use in an inductive coupler for downhole drilling components," U. S. Patent: 7098767 B2, 2006.

[12] J. Fox, J. Miller, D. R. Hall et al., "Transmitting data through a downhole environment,” U. S. Patent: 7298287 B2, 2007. 
[13] J. Fox and D. R. Hall, "Element for use in an inductive coupler for downhole components," U. S. Patent: 7511598 B2, 2009.

[14] D. R. Hall, T. Hall, C. Sneddon et al., "Inductive coupler for downhole components and method for making same," U. S. Patent: 7116199 B2, 2006.

[15] J. Fox and D. R. Hall, "Polished downhole transducer having improved signal coupling," U. S. Patent: 7019665 B2, 2006.

[16] D. Wall, T. Hassell, T. Christiansen et al., "Inductive coupler assembly for downhole transmission line," U. S. Patent 10612318 B2, 2020.

[17] H. Sun, "Technology of magnetic induction transmission of intelligent drill pipe and its channel characteristics," Journal of China University of Petroleum, vol. 37, no. 06, pp. 172-176, 2013.

[18] Z. Li, Z. Liu, F. Hou et al., "Design and simulation of drill pipe coupler with magnetic induction transmission," Sino-Global Energy, vol. 21, no. 06, pp. 55-59, 2016.

[19] Y. Hu, Y. Huang, and L. Shi, "Channel modeling and simulation of high-frequency magnetic coupling wired drill pipe," Acta Petrolei Sinica, vol. 39, no. 11, pp. 1292-1298, 2018.

[20] A. Baccigalupi, P. Daponte, and D. Grimaldi, "On a circuit theory approach to evaluate the stray capacitances of two coupled inductors," IEEE Transactions on Instrumentation and Measurement, vol. 43, no. 5, pp. 774-776, 1994.

[21] J. Biernacki and D. Czarkowski, "High frequency transformer modeling," in Proceedings of the IEEE International Symposium on Circuits and Systems, pp. 676-679, IEEE, Daegu, South Korea, May 2001.

[22] J. H. Harlow, Electric Power Transformer Engineering, Taylor \& Francies, Oxfordshire UK, 2007.

[23] Y. Xu, H. Zhang, and Z. Guan, "Dynamic characteristics of downhole bit load and analysis of conversion efficiency of drill string vibration energy," Energies, vol. 14, no. 1, 2021.

[24] Y. L. Bihan, "Study on the transformer equivalent circuit of eddy current nondestructive evaluation," NDT and E International, vol. 36, no. 5, pp. 297-302, 2003.

[25] R. R. Hughes and S. Dixon, "Analysis of electrical resonance distortion for inductive sensing applications," IEEE Sensors Journal, vol. 18, no. 14, pp. 5818-5825, 2018.

[26] J. Jia, Y. Su, Y. Shen et al., "Signal transmission capability improvement of a telemetry drill string using the reflected signal at the transmission line termination," Mathematical Problems in Engineering, vol. 2020, Article ID 7391530, 11 pages, 2020. 